${ }^{1}$ ГБУЗ «Санкт-Петербургский клинический научнопрактический центр специализированных видоп медицинской помощи (онкологический)" (Санкт-Петербург, Россия)

${ }^{2}$ СЗГМУ им. И.И. Мечникова (Санкт-Петербург, Россия)

\section{CANCER MOONSHOT}

\author{
Ф.В. Моисеенко ${ }^{1,2}$, В.М. Моисеенко ${ }^{1}$
}

\section{CANCER MOONSHOT}

Ф.В. Моисеенко ${ }^{1,2}$ Доктор медицинских наук, Санкт-Петербургский клинический научно-практический центр специализированных видов медицинской помощи (онкологический), СЗГМУ им. И.И. Мечникова, 197758, Россия, Санкт-Петербург, пос. Песочный, ул. Ленинградская, д. 68А, лит. А. Teл.: 8 (812) 573-9191.E-mail: moiseenkofv@gmail.com.

В.М. Моисеенко ${ }^{1}$ Доктор медицинских наук, профессор, директор, Санкт-Петербураский клинический научно-практический центр спещиализированных видов медицинкой помощи (онкологический), 197758, Россия, Санкт-Петербург, пос. Песочный, Ленинградская ул., д. 68А, лит. А.

F.V. Moiseenko ${ }^{1,2}$ Doctor of Medicine, St. Petersburg Clinical Research Center of specialized types of care (Oncology), North-Western State Medical University named after I.I. Mechnikov, 197758, Russia, St. Petersburg, Leningradskaya st., 68A, lit. A. Phone: 8 (812) 573-9191. E-mail: moiseenkofv@gmail.com.

V.M. Moiseyenko ${ }^{1}$

Doctor of Medicine, Professor, Director, St. Petersburg Clinical Research Center of specialized types of care (Oncology), 197758, Russia, St. Petersburg, pos. Pesochniji, Leningradskaya ul., 68A, lit. A.

В данной статье рассматривается инициированная в 2016 году правительством США программа Cancer Moonshot. Программа ставит амбициозную цель пройти 10-летний путь изучения рака за 5 лет и «искоренить рак, таким как мы его знаем». Для достижения поставленной цели группой из 150 выдающихся ученых и врачей были сформулированы направления, значимые достижения в которых должны изменить существующую ситуацию. Среди них облегчение доступа к клиническим исследованиям и расширение сети обмена клиническими и научными данными, активное исследование иммунотерапевтических подходов, изучение механизмов резистентности, симптоматическая терапия и некоторые другие. Обзор посвящен детальному рассмотрению каждого из пунктов предлагаемой программы.

ключевые слова: рак, лечение злокачественных опухолей, инноващии, перспективные исследования.

This article discusses the Cancer Moonshot program launched in 2016 by the government of US. The main purpose of this program is to «...end cancer as we know it...». In order to achieve this aim 150 outstanding scientists and oncologists formulated 7 main recommended fields of research, breakthrough in which should change cancer paradigm. Among them network for direct patient engagement into clinical trials and high-throughput molecular research, cancer immunotherapy translational science research, drug therapy resistance mechanisms elucidation, symptom management and others. Here we try to show the details of each recommendation.

Keywords: cancer, solid tumors treatment, innovations, perspective research. 
$\mathrm{B}$ 1971 году президент США Ричард Никсон провозгласил «войну с раком». Это было закреплено на уровне федерального акта. Тем не менее, к 2006 году, несмотря на значительные успехи в лечении некоторых опухолей, прогресс в целом оценивался как недостаточный. До 2016 года было предпринято несколько попыток обновления акта, направленных на его оптимизацию с учетом новых данных о патогенезе и принципах лечения злокачественных опухолей.

В 2016 году вице-президент США Джо Байден предложил новую программу, названную Cancer Moonshot. Целью этой программы стало «искоренить рак, таким как мы его знаем» («...end cancer as we know it...»). Существенным отличием новой программы явилось тщательное планирование направлений деятельности, привлечение к формированию задач 150 выдающихся онкологов современности, а также направленность на максимально возможное объединение не только крупных научных центров, но также и обычных клинических подразделений. Ниже мы приводим основные планируемые направления, существенные достижения по которым должны изменить ситуацию со злокачественными опухолями в течение 5 лет.

\section{Сеть для прямой и быстрой идентификации и включения пациентов в клинические исследования (Network for Direct Patient Engagement)}

Крупные исследования последних десятилетий однозначно свидетельствуют о значительной гетерогенности злокачественных опухолей на молекулярном уровне [14]. Основным следствием этого является нецелесообразность в проведении одинакового лечения больным даже с опухолями одного типа. Многочисленные работы, сравнивавшие различные варианты цитостатической и таргетной терапии, позволили установить, что у отдельных больных лечение может позволить достичь существенных изменений общей выживаемости, значительного уменьшения опухолевой массы или способствовать излечению, в тоже время у других пациентов с неотличимыми на основании клинических признаков опухолями аналогичное лечение будет абсолютно неэффективным. Существенные сдвиги в понимании патогенетической гетерогенности были сделаны после выявления различных активирующих мутаций [12]. Это наиболее ярко проявилось при такой химиорезистентной опухоли как немелкоклеточный рак легкого, когда для относительно небольшой группы больных было установлено основное патогенетическое нарушение, определяющее деление и пролиферацию опухолевых клеток - активирующие мутации (в том числе EGFR, ALK, ROS1 и др.) [16]. Применение специфических таргетных препаратов при каждом из видов молекулярных нарушений позволяет достичь выраженного клинического эффекта и существенных улучшений показателей выживаемости [18]. Однако широкое при- менение высокоэффективных таргетных препаратов осложняется относительно узкой группой больных с каждым из видов известных активирующих мутаций. Данный факт, кроме сложной логистической системы их идентификации, также требует несоизмеримо больших усилий по определению оптимального лечебного подхода. Проведение крупных клинических исследований, являющихся единственным на настоящий момент стандартным подходом для подтверждения эффективности того или иного лекарственного препарата в конкретной группе пациентов с наличием предиктивного фактора, крайне затруднительно в связи с редкостью отдельных типов молекулярных нарушений.

Один из возможных путей решения этой проблемы является формирование предложенной в рамках программы Cancer Moonshot сети баз данных молекулярно- и клинически охарактеризованных опухолей. В рамках этой задачи предполагается проспективно идентифицировать пациентов, пожелавших принять участие в работе, и подвергать их опухоли максимальному существующему на настоящий момент спектру методов молекулярного профилирования. На сегодняшний день в связи со стоимостью и технической сложностью методы молекулярно-генетического анализа с охватом всего генома могут быть предложены крайне ограниченному кругу пациентов даже в США, несмотря на несравнимо более высокий уровень развития требуемой инфраструктуры относительно прочих регионов. Формирование крайне сложной с логистической точки зрения базы данных, с одной стороны обеспечивающей учет максимального числа клинических характеристик, регистрацию результатов проспективного проведения сложнейших молекулярно-генетических исследований, а с другой позволяющую больным динамически получать информацию о специфических генетических нарушениях, делающих опухоли потенциально чувствительными к отдельным препаратам, может позволить существенно увеличить возможности включения больных с редкими нарушениями в клинические исследования. В свою очередь, большее число больных с каждым из видов мутаций позволит значимо ускорить клиническое изучение и регистрацию наиболее эффективных из лекарственных средств.

Кроме того, в случае желания пациента, программа будет позволять выполнять последовательные биопсии на нескольких этапах клинического течения болезни: при достижении эффекта, после формирования резистентности. Практически наверняка данная опция будет доступна далеко не для всех больных, в связи с, например, полным исчезновением очагов или ухудшением состояния больного при развитии резистентности. Тем не менее, даже ограниченное число подобных больных, учитывая масштабы проекта, значительно расширит представления о механизмах эффективности и резистентности к каждому из препаратов. 


\section{Организация предклинического и клинического изучения иммунотерапии злокачественных опухолей (Cancer Immunotherapy Translational Science Network)}

B XIX века иммунотерапия была идентифицирована как один из возможных эффективных методов борьбы со злокачественными новообразованиями [7]. Ранние работы Коли показали потенциальную возможность формирования противоопухолевого иммунитета за счет экспозиции организма к отдельным видам инфекционных патогенов [13]. Кроме того, достоверно зарегистрированные в клинической практике случаи спонтанных регрессов диссеминированных опухолей различного гистологического происхождения на протяжении всего этого времени постоянно «подогревали» интерес к этой области, так как не могли получить другого объяснения кроме внезапной активации иммунной системы против опухолевых клеток.

Тем не менее, в течение многих последующих десятилетий данный метод не смог привести к стабильному результату у значимого числа пациентов. Большинство работ, проведенных в прошлом веке, были направлены на формирование (дендритноклетоные вакцины) или стимулирование уже существующего (интерфероны, интерлейкины) иммунитета для реализации его противоопухолевого действия.

Существенные ограничения на возможность создания стандартизованного метода иммунотерапии накладывали несколько факторов. Во-первых, отсутствие в течение более чем 50 лет адекватной модели для изучения взаимодействия иммунитета и опухолевых клеток у лабораторных животных [10, 20]. Сложность этого механизма подтверждается ложным представлением об отсутствии иммуногенности опухолевых клеток, доминировавшим в течение длительного времени. Данный постулат был достоверно опровергнут лишь в 60-х годах прошлого столетия [3]. Во-вторых, крайняя технологическая сложность и индивидуальность каждого из продуктов (вакцина на основе дендритных клеток). Высокая стоимость производства, условия GMP, использование индивидуального клеточного материала делала невозможным идентичное повторение продуктов, полученных на предыдущих этапах исследований, что в свою очередь исключало возможность проведение широких рандомизированных исследований. Кроме того, необходимость работы с клеточными продуктами, а также отсутствие четких детерминант появление активных против опухолевых клеток клонов Т-лимфоцитов не позволяла на протяжении длительного времени стандартизировать методики их приготовления.

Несмотря на все изложенные выше сложности, в 90-х годах XX века, в частности в работах Джима Элисона - одного из основоположников и перво- открывателей механизмов взаимодействия иммунитета и опухоли, были представлены веские доказательства не только существования противоопухолевого иммунитета, но также и присутствия ингибирующего влияния опухолевых клеток на уже сформированный иммунитет. Довольно длительное изучение механизмов, тормозящих реализацию противоопухолевого действия иммунной системы, позволили идентифицировать большое число молекул, участвующих в этом процессе, как со стороны опухолевых, так и иммунокомпетентных клеток. Любопытно обратить внимание читателя на тот факт, что большинство открытых на сегодняшний момент механизмов активных при опухолевых заболеваниях являются естественными методами контроля организма за активностью иммунологических реакций. Так, фермент IDO (индоламин 2,3-диоксигеназа) является ключевым иммуносупрессивным фактором, предотвращающим активацию иммунитета матери против плода [6].

После идентификации первых подобных механизмов: взаимодействия CTLA-4 и PD-1 с лигандом PD-L1 на опухолевых, дендритных клетках и лимфоцитах были созданы лекарственные препараты, которые буквально изменили стандарты лечения злокачественных опухолей. Принципиально новый механизм действия позволил предположить отсутствие перекрестной резистентности с цитостатический и таргетной терапией, отличный от известных ранее профиль токсичности, связанный с развитием неконтролируемых воспалительных реакций в различных органах, также позволяет комбинировать новые иммунотерапевтические препараты со стандартными методами. Все сказанное выше привело к тому, что буквально за несколько лет в рамках клинических исследований была доказана эффективность ингибиторов сигнальных точек (check-point inhibitors) для более 20 нозологических форм, а для 17 к 2016 году были получены регистрационные удостоверения FDA. К сожалению, к настоящему моменту можно однозначно сказать, что первое поколение подобных препаратов, к которым относятся ингибиторы CTLA-4 (ипилимумаб, тремелимумаб) и PD-1 $\backslash \mathrm{PD}-\mathrm{L} 1$, объединенные в одну группу, несмотря на различные точки реализации эффекта, в связи с практически идентичными клиническими результатами (ниволюмаб, пембролизумаб, атезолизумаб, авелумаб, а также первый отечественный ингибитор - BCD-100) позволяют добиться клинически значимого противоопухолевого эффекта не более чем у 10-20\% пациентов. Только в отдельных группах частота объективных ответов превышает 50-60\%, например, резистентной лимфоме Ходжкина, колоректального рака с микросателитной нестабильностью. Объяснением этому могут быть несколько: во-первых, ингибирующее влияние опухоли на иммунокомпетентные клетки не ограничивается взаимодействием PD-1 и PD-L1, a во-вторых, реализация противоопухолевого эффекта 
ингибиторов контрольных точек требует не только активности механизма блокировки на опухолевых клетках, но также и присутствия активированных против опухолевых клеток Т-лимфоцитов. Можно с уверенностью сказать, что потенциал противоопухолевого действия иммунной системы изучен далеко не полностью. Для получения максимального эффекта требуется создание моделей, которые в более полной мере будут отражать реальную ситуацию взаимодействия иммунной системы и опухолевых клеток, которую мы наблюдаем у пациентов. Кроме того, для достижения максимального результата, который в случае злокачественных опухолей будет реализован в полном уничтожении опухолевых клеток, требуется создание уникальных, значительно более продуктивных, информативных технологий визуализации, генетического и протеомного анализа, а также существенная оптимизация методов обработки огромных наборов данных, получаемых при исследовании сложных биологических систем.

Отдельным фактором, который, несмотря на поистине гигантские усилия, остается пока за гранью нашего понимания, является удивительно различная клиническая эффективность. Так, например, при меланоме, у части пациентов наблюдаются длительные, находящиеся на грани критериев излечения, выраженные эффекты, в тоже время у большего числа пациентов болезнь продолжает прогрессировать на фоне проводимой иммунотерапии. Наиболее активно внедряемым маркером является экспрессия PD-L1 [11]. Селекция пациентов на основании высокой экспрессии этой молекулы позволяет существенно повысить частоту объективных эффектов [15]. В тоже время многие характеристики этого маркера, среди которых количественный, а не качественный характер анализа, динамическое изменение его значений во времени и, чаще всего, независимо от проводимых противоопухолевых мероприятий, не позволяют рассматривать его как более или менее окончательный вариант.

Таким образом, для успешного, а главное быстрого улучшения нашего понимания иммуноопосредованных механизмов взаимодействия организма и опухоли требуется максимальное объединение усилий крупных научных и клинических центров, облегчение доступа к новым данным среди участников консорциума, стандартизация протоколов создания тех или иных биологических препаратов.

\section{Идентификация терапевтических мишеней для преодоления резистентности (Therapeutic Target Identification to Overcome Drug Resistance)}

Не секрет, что для локализованных стадий уничтожение опухолевых клеток в ряде случаев может быть достигнуто с помощью локальных методов (хирургического и лучевого), эффективность которых практически не зависит от молекулярной гетерогенности удаляемого образования. В свою очередь лекарственные средства, взаимодействуя в течение определенного периода с отдельными (не всегда идентифицированными) элементами опухолевого патогенеза, должны также вызывать гибель всех злокачественных клеток тем или иным способом, что теоретически равносильно излечению. По различным причинам применение лекарственной терапии крайне редко позволяет вылечить онкологических пациентов. Сохранившиеся на фоне первичного лечения клетки продолжают эволюционировать с появлением и/или увеличением пропорции резистентных клонов, что на клиническом уровне означает прогрессирование болезни и рано или поздно приводит к гибели пациента. В этой связи, формирование междисциплинарного инициативного проекта, направленного на идентификацию чувствительных «точек» опухолевых клеток, которые в свою очередь могут быть использованы для создания новых лекарственных препаратов, способных предотвратить или преодолеть способность опухолевых клеток становиться нечувствительными к проводимому лечению, является высокоактуальным.

Понимание причин и времени возникновения резистентности, выявление пациентов, имеющих повышенный риск ее формирования, должно в существенной мере изменить наши подходы к лечению злокачественных неопластических процессов.

К сожалению, процесс формирования резистентности является крайне сложным [9]. При этом часть опухолей имеют черты первичной резистентности или нечувствительности к различным вариантам терапии, а другая часть может приобретать их в ходе лечения, несмотря на первичный ответ [9]. Механизмы, определяющие эти различия, изучены несмотря на болышое число исследований в этом направлении недостаточно. Тем не менее, на сегодняшний момент очевидно, что формирование резистентности определяется большим числом различных факторов, некоторые из которых могут присутствовать у отдельных пациентов одновременно. Кроме того, механизмы резистентности изменяются и эволюционируют с течением времени на фоне проводимой терапии, что обеспечивает опухолевым клеткам постоянное преимущество в выживаемости.

Подобный проект в рамках инициативы Cancer Moonshot будет интегрировать молекулярный анализ образцов опухолевой ткани, полученных до и после лечения, по факту рецидива заболевания и при формировании резистентности. При этом планируется охватить максимально возможное число вариантов злокачественных опухолей и все существующие методы лечения, включая стандартную химиотерапию, лучевую терапию, таргетную и иммунотерапию. 


\section{Национальная система обмена и анализа данных анализа опухолей (A National Cancer Data Ecosystem for Sharing and Analysis)}

На сегодняшний момент развитие онкологии, ее направленное на излечение движение во многом зависит от анализа крупных и различных по своей форме и сути наборов данных, производимых in silico и другими технологиями. Огромное количество подобных данных продуцируется ежедневно как в области предклинических исследований, так и в клинических испытаниях. На этом фоне недостаток координированной работы в области анализа получаемых данных существенно ограничивает движение онкологической науки. Скоординированный анализ идентификации и применения взаимосвязей между данными молекулярно-генетических исследований (в том числе полногеномного анализа индивидуальных образцов и модельных систем) и клиническими результатами проводимых воздействий (клинический ответ на терапию, особенности характеристик пациентов) имеют критическое значение дня разработки новых методов противоопухолевого воздействия. В этой связи нельзя не вспомнить тот факт, что наиболее яркие открытия в области таргетной терапии были получены не в результате целенаправленного поиска тех или иных нарушений, а в результате анализа клинических образцов с зарегистрированным ответом на конкретный препарат. Именно таким образом была выявлена взаимосвязь между активирующими мутациями EGFR и чувствительностью к низкомолекулярным ингибиторам этого рецептора, транслокациями ALK и ROS1 и эффективностью кризотиниба $[2,4,19]$.

Другим фактором, определяющим острую необходимость облегчения доступа к медицинской и биологической информации пациентов, является широкий спектр методик, которые применяются для лечения больных с онкологическим заболевание на сегодняшний день. Зачастую лечение инициируется в одном медицинском учреждении, молекулярногенетический анализ опухолевых образов производиться в другом, а различные методы локального воздействия, среди которых радиохирургические методы воздействия, в третьем. Кроме того, большое число клинических исследований новых препаратов, производимых в различных центрах, требуют быстрого и скоординированного обмена медицинской информацией для обеспечения максимально эффективного варианта терапии для отдельно взятого пациента.

Для достижения существенного прорыва в этой области, в рамках программы Cancer Moonshot планируется создать цельную экосистему обмена данными, что потребует не только унификации форматов баз данных на уровне in silico, но также и тщательную проработку логистики выполнения аналитических методов.

\section{Исследования в области симптоматической терапии (Symptom Management Research)}

Симптоматическая терапия является неотъемлемым элементом лечения пациентов со злокачественными опухолями. Ежегодно в США более 650000 пациентов получают тот или иной вариант лекарственной терапии, а 470000 проводится лучевая терапия, в том числе в комбинации с системным воздействием. К сожалению, появление симптомов заболевания, определяющих качество жизни больных, определяется не только самим заболеванием, но также и проводимыми лечебными мероприятиями. Около трети пациентов отмечают появление от одного до трех нежелательных явлений выраженной степени. Эти симптомы влияют на снижение функционального статуса и существенно снижают качество жизни пациентов, а зачастую требуют госпитализации и представляют угрозу для жизни. В свою очередь наличие подобных проявлений требуют перерывов в лечении, что может негативно отражаться на его эффективности. При этом за кадром изучения остается влияние активного контроля симптомов заболевания и нежелательных явлений лечения на продолжительность жизни пациентов. Так, в одном из исследований при не мелкоклеточном раке легкого было показано, что активная симптоматическая помощь статистически достоверно увеличивает общую выживаемость пациентов с этим заболевание независимо от противоопухолевых мероприятий [21].

Активное применение методов регистрации и контроля симптомов на фоне течения злокачественного неопластического процесса позволит ускорить разработку системы симптоматической помощи для всех известных злокачественных опухолей. Такая система позволит облегчить и ускорить связь между пациентами, их семьями и специалистами по каждой отдельно взятой проблеме, а также разработать адекватные информационные ресурсы для самообразования больных. В свою очередь уменьшение страданий, связанных с проявлениями симптомов, должно однозначно положительно отразиться как на отдаленных результатах лечения, так и на качестве жизни на фоне его проведения.

Для достижения поставленной цели планируется существенно пересмотреть и переработать рекомендации по симптоматической терапии. Сформировать систему контроля за сообщением о нежелательных явлениях и их динамики на фоне проводимых мероприятий по их коррекции. Определить наиболее эффективные пути и обеспечить их внедрение в рамках системы медицинской помощи в масштабах страны. 


\section{Профилактика и раннее выявление: внедрение evidence-based подходов (Prevention and Early Detection: Implementation of Evidence-Based Approaches)}

Несмотря на успехи в лечении злокачественных опухолей, которые в существенном проценте случаев позволяют вылечить пациентов, применение современных методов удаления опухолевых клеток чаще всего несет калечащий характер и представляет угрозу жизни пациента сами по себе. Кроме того, большинство из известных методов воздействия на опухоль имеют крайне высокую стоимость, что с учетом распространенности злокачественных опухолей создает значимую финансовую нагрузку на мировые системы здравоохранения. При этом уже сейчас некоторые виды злокачественных опухолей можно предотвратить. Например, полномасштабное применение разработанных и доказанных методов скрининга поможет предотвратить 90\% смертей, связанных с раком шейки матки, $70 \%$ - с раком толстого кишечника и $90 \%$ - с раком легкого.

Другой проблемой в области снижения смертности от злокачественных опухолей является недостаточно широкое использование методов генетической диагностики наследственных форм опухолей. На сегодняшний момент известно более 50 генетических нарушений, имеющих наследственный характер, которые определяют повышенную вероятность развития опухолей у их носителей и являются по существу единственным известным значимым фактором риска. Важным шагом к решению этой проблемы станет формирование разветвленной системы тестирования наследственных факторов, разработка и внедрение оптимальных стратегий для более раннего выявления опухолей у носителей наследственных нарушений. Например, для синдрома Линча, у носителей которого значимо повышена вероятность возникновения рака толстой кишки и эндометрия, частота идентификации молекулярных нарушений по данным США не превышает $5 \%$.

На настоящий момент разработаны и готовы к клиническому применению несколько вакцин против онкогенных штаммов ВПЧ, который определяет этиопатогенез практически всех опухолей шейки матки, вульвы, вагины, полового члена и орофарингеальной зоны. Тем не менее, частота вакцинирования даже на территории США не превышает 40\% у девочек и $22 \%$ - у мальчиков. Сходная ситуация наблюдается и с раком толстой кишки, при котором своевременное выявление и удаление доброкачественных новообразований в ходе колоноскопии позволяет предотвратить большую часть опухолей этой локализации. При этом треть всего населения США в возрасте от 50 до 75 лет не проходит необходимый минимум скрининга.
Отдельную роль играет снижение частоты табакокурения, которое позволяет значительно снизить не только заболеваемость и смертность раком легкого, но также и сердечнососудистыми заболеваниями.

К сожалению, широкое применение высокоэффективной вакцинопрофилактики и снижение табакокурения оставляет желать лучшего. Для решения этой проблемы требуется активное изучение методов применения данных направлений. Несмотря на то, что генетические методы диагностики наследственных мутаций, ассоциированных с развитием опухолей, широкодоступны, их стоимость по-прежнему остается крайне высокой, что значительно снижает возможности их оптимального применения.

\section{Ретроспективный анализ биообразцов больных, получивших стандартные варианты лечения (Retrospective Analysis of Biospecimens from Patients Treated with Standard of Care)}

Уже на протяжении многих лет сотням тысяч пациентов по всему миру проводится стандартная терапия. У некоторых из них удается достичь значимого увеличения продолжительности жизни, а в редких случаях и излечения. Так, в качестве примера можно привести исследование N9741 при метастатическом колоректальном раке, который однозначно рассматривается сейчас как инкурабельное заболевание. Из 1508 включенных пациентов с неоперабельным раком толстой кишки, полный регресс опухолевых очагов с помощью лекарственной терапии был достигнут у 62 больных. В течение последующих 10 лет наблюдения у 10/62 пациентов прогрессирования опухолевого процесса не произошло, что позволяет считать их излеченными [8]. В тоже время у большей части наблюдается прогрессирование и гибель.

В некоторых случаях рецидив заболевания, возникший после первичного лечения, за короткое время уносит жизнь пациента. А иногда повторное использование первичных режимов после иммунотерапии больным, не ответившим на лечение ранее, позволяет добиться выраженных клинических эффектов. Что лежит в основе разной эффективности стандартной терапии к настоящему моменту не установлено. Причинами тому являются крайняя сложность определяющих патогенез опухолей молекулярных механизмов, значительная гетерогенность самого опухолевого процесса, а также разобщенность подобных случаев уникальной эффективности. Наряду с этим, в то время как разработку и внедрение новых лекарственных препаратов активно поддерживают фармацевтические компании, на изучение механизмов эффективности препаратов уже вошедших в клиническую практику выделяется значительно меньше ресурсов.

На настоящий момент причины, почему один вид лечения может иметь совершенно различные 
результаты у больных с одним видом опухоли и одинаковыми клиническими характеристиками, являются загадкой. При этом очевидно, что решение этой проблемы позволит спасти большое число жизней. Тщательный и глубокий анализ архивных материалов с целью определения генетических, клеточных, иммунных и других событий, которые определяют эффект от лечения у одних пациентов и резистентность у других позволит создать субклассификацию опухолей на молекулярном уровне. Кроме того, данный подход поможет идентифицировать пациентов, оптимальным лечением для которых является стандартная лечебная тактика, и других - требующих для достижения аналогичного результата применения новых или дополнительных методов. Также нельзя в этой связи не обратить внимания на проблемы перелечивания - выделение подгруппы, не требующей дополнительных лекарственных воздействий после радикального хирургического лечения, избавит их от ненужной токсичности, а систему здравоохранения от избыточных затрат.

\section{Фузионные онкопротеины при злокачественных опухолях у детей (Fusion Oncoproteins in Childhood Cancers)}

Несмотря на существенные успехи в педиатрической онкологии, злокачественные опухоли остаются одной из основных причин смерти удетей. Существенный отпечаток также накладывает непосредственная и отсроченная токсичность проведенного лечения. Для детских опухолей характерно наличие специфических генетических нарушений - транслокаций. Наличие этих нарушений часто определяет клинические особенности конкретного опухолевого процесса (например, EWS-FL1 при саркоме Юинга, PAX-FOXO при альвеолярной рабдомиосаркоме, C11orf95-RELA при супратенториальной эпендимоме). Эти транслокации приводят к формированию, так называемых фузионных белков, которые становятся онкогенными. Фузионные белки широко известны как драйверные нарушения при отдельных опухолях с низким числом прочих соматических мутаций. Поэтому они представляют крайне перспективную мишень для потенциального терапевтического воздействия.

Недавние исследования показали, что многие фузионные белки реализуют свою функцию через дерегуляцию белковых комплексов, которые контролируют экспрессию других генов или статус хроматина. К сожалению, более глубокое изучение фузионных белков, необходимое для создания эффективных лекарственных препаратов крайне затруднено. Вопервых, число предклинических моделей детских опухолей существенно ограниченно. А во-вторых, крайне недостаточно систематических данных о молекулярных и эпигенетических характеристиках данных новообразований.
Несмотря на то, что отдельные исследовательские группы активно изучают фузионные белки как мишени для противоопухолевой терапии, очевидно, что идентификация ключевых взаимосвязей требует более широкого и системного подхода к созданию клеточных моделей, сбору, анализу и хранению данных о получавших лечение пациентах. Кроме прочего, детские опухоли относятся к редким опухолям, что, вне всякого сомнения, на этапе перехода от биологического анализа к попытками терапевтического воздействия потребует высоко динамичной и организованной системы клинического исследования әффективности.

В рамках программы Cancer Moonshot планируется разработать полноценную коллекцию генетически охарактеризованных клеточных линий, мышиных моделей и индуцированных плюрипотентных стволовых клеток с наличием фузионных белков, которые на настоящий момент отсутствуют. Расширить и углубить понимание механизмов, через которые фузионные белки реализуют свою патологическую функцию. Определить ключевые уязвимые точки опухолей, индуцированных появлением фузионных белков, за счет функционального геномного скрининга и создания карт ключевых функционально активных доменов для каждого фузионного онкопротеина. Разработать новые низкомолекулярные препараты для исследования и определения эффективности на мышиных моделях.

\section{Формирование атласов опухолей человека (Generation of Human Tumor Atlases)}

Целью этого этапа является формирование атласов детских и взрослых опухолей путем документирования генетических нарушений и молекулярных каскадов, а также клеточных взаимодействий, которые определяют формирование и развитие опухолей от предопухолевых очагов до первичных опухолей, метастастазирования и приобретения резистентности. Данные атласы должны включать различные популяции больных и интегрировать новые и уже существующие наборы данных для всестороннего описания молекулярных, клеточных и физиологических событий, ассоциированных с отдельными опухолевыми клетками, опухолевыми очагами в целом, первичной ткани и очагами метастазирования. Создание точных карт динамической архитектоники отдельных опухолей во времени позволит значительно лучше понять эволюцию опухолей, а также механизмы сложнейших взаимодействий с нормальными клетками, лекарственными препаратами и иммунокомпетентными клетками организма. Формирование подобных атласов потребует тщательной работы междисциплинарной команды по сбору последовательных образцов из первичной 
опухоли и метастазов, аннотации клинической информации.

Надо сказать, что данное направление является продолжением уже инициированной работы в рамках TCGA (Tumor Cancer Genome Atlas). В рамках этого проекта были получены принципиальные данные о классификации опухолей различного происхождения, новых молекулярных нарушений, воздействие на которые может привести к выраженному противоопухолевому эффекту $[1,5,17]$. Так, были проанализированы опухоли әндометрия, желудка, легкого, головы и шеи и многие другие.

\section{Разработка новых технологий определения рака (Development of New Enabling Cancer Technologies)}

Формирование инновационных технологий в области визуализации, инструментальных методов диагностики (многомерная визуализация на клеточном уровне, молекулярно-генетические технологии и методы микродозовой внутриопухолевой доставки), биологических моделей (на основании первичных клеточных культур пациентов, гуманизированных мышиных моделей), компьютерных платформ, которые позволят объединить молекулярную, клеточную, тканевую структуру и клиническую информацию об опухоли. Тестирование отдельных потенциально эффективных препаратов на ранних этапах клинического изучения обычно проводится на клеточных линиях или генетически модифицированных мышиных моделях, которые фокусируются на отдельных или узких опухолеассоциированных мутациях. Несмотря на то, что эти модели имеют большое значение для изучения биологических механизмов, они малоприменимы для реальной практики. В связи с этим, создание 3-D моделей, отражающих оригинальную архитектонику и сложность молекулярной структуры первичной ткани, по мнению әкспертов, представляется наиболее перспективным.

\section{Заключение}

Все сказанное выше определяет основные направления для изменения ситуации в онкологии. Несмотря на громкие заявления и амбициозность ожидаемых результатов, оценить программу иначе как крайне положительно нельзя. Вне всякого сомнения, серьезные усилия, в том числе на государственном уровне, направленные на максимально возможное объединение усилий крупных научных учреждений, должны привести к значительным результатам. Кроме того, даже если проблему рака не удастся решить в отведенные сроки, подобные проекты внесут колоссальный вклад в наши представления о биологии опухолевого роста, позволят вооружить онкологов новыми высокоэффективными методами лечения и послужат неоценимой школой для молодых ученых и врачей.

\section{Список литературы}

1. Bass A.J., Thorsson V., Shmulevich I., Reynolds S.M. Comprehensive molecular characterization of gastric adenocarcinoma. // Nature. - 2014. - Vol. 513(7517). P. 202-209.

2. Bergethon K., Shaw A.T., Ou S.H., Katayama R., Lovly C.M., McDonald N.T., Massion P.P., Siwak-Tapp C., Gonzalez A., Fang R., Mark E.J., Batten J.M., Chen H., Wilner K.D., Kwak E.L., Clark J.W., Carbone D.P., Ji H., Engelman J.A., Mino-Kenudson M., Pao W., Iafrate A.J. ROS1 rearrangements define a unique molecular class of lung cancers. // J Clin Oncol. - 2012. - Vol. 30(8). P. 863-870.

3. Burnet M. Cancer; a biological approach. I. The processes of control. // Br Med J. - 1957. - Vol. 1(5022). P. 779-786.

4. Camidge D.R., Bang Y.J., Kwak E.L., Iafrate A.J., Varella-Garcia M., Fox S.B., Riely G.J., Solomon B., Ou S.H., Kim D.W., Salgia R., Fidias P., Engelman J.A., Gandhi L., Janne P.A., Costa D.B., Shapiro G.I., Lorusso P., Ruffner K., Stephenson P., Tang Y., Wilner K., Clark J.W., Shaw A.T. Activity and safety of crizotinib in patients with ALK-positive non-small-cell lung cancer: updated results from a phase 1 study. // Lancet Oncol. - 2012. - Vol. 13(10). P. 1011-1019.

5. Cerami E., Demir E., Schultz N., Taylor B.S., Sander C. Automated network analysis identifies core pathways in glioblastoma. // PLoS One. - 2010. - Vol. 5(2). P. e8918.

6. Chen W. IDO: more than an enzyme. // Nat Immunol. - 2011. - Vol. 12(9). P. 809-811.

7. Couzin-Frankel J. Breakthrough of the year 2013. Cancer immunotherapy. // Science. - 2013. - Vol. 342(6165). P. $1432-1433$.

8. Goldberg R.M. N9741: a phase III study comparing irinotecan to oxaliplatin-containing regimens in advanced colorectal cancer. // Clin Colorectal Cancer. - 2002. - Vol. 2. P. 81.

9. Gonzalez-Angulo A.M., Morales-Vasquez F., Hortobagyi G.N. Overview of resistance to systemic therapy in patients with breast cancer. // Adv Exp Med Biol. - 2007. - Vol. 608. P. 1-22. 
10. Hunig T. T-cell function and specificity in athymic mice. // Immunol Today. - 1983. - Vol. 4(3). P. 84-87.

11.Johnson D.B., Peng C., Sosman J.A. Nivolumab in melanoma: latest evidence and clinical potential. // Ther Adv Med Oncol. - 2015. - Vol. 7(2). P. 97-106.

12. Lynch T.J., Bell D.W., Sordella R., Gurubhagavatula S., Okimoto R.A., Brannigan B.W., Harris P.L., Haserlat S.M., Supko J.G., Haluska F.G., Louis D.N., Christiani D.C., Settleman J., Haber D.A. Activating mutations in the epidermal growth factor receptor underlying responsiveness of non-small-cell lung cancer to gefitinib. // N Engl J Med. 2004. - Vol. 350(21). P. 2129-2139.

13. McCarthy E.F. The toxins of William B. Coley and the treatment of bone and soft-tissue sarcomas. // Iowa Orthop J. - 2006. - Vol. 26. P. 154-158.

14. McGranahan N., Swanton C. Clonal Heterogeneity and Tumor Evolution: Past, Present, and the Future. // Cell. - 2017. - Vol. 168(4). P. 613-628.

15. Reck M., Rodríguez-Abreu D., Robinson A.G., Hui R., Csöszi T., Fülöp A., Gottfried M., Peled N., Tafreshi A., Cuffe S., O'Brien M., Rao S., Hotta K., Leiby M.A., Lubiniecki G.M., Shentu Y., Rangwala R., Brabmer J.R., KEYNOTE-024 I. Pembrolizumab versus Chemotherapy for PD-L1-Positive Non-Small-Cell Lung Cancer. // N Engl J Med. - 2016. Vol. 375(19). P. 1823-1833.

16. Rolfo C., Giovannetti E., Hong D.S., Bivona T., Raez L.E., Bronte G., Buffoni L., Reguart N., Santos E.S., Germonpre P., Taron M., Passiglia F., Van Meerbeeck J.P., Russo A., Peeters M., Gil-Bazo I., Pauwels P., Rosell R. Novel therapeutic strategies for patients with NSCLC that do not respond to treatment with EGFR inhibitors. // Cancer Treat Rev. - 2014. - Vol. 40(8). P. 990-1004.

17. Rooney M., Devarakonda S., Govindan R. Genomics of squamous cell lung cancer. // Oncologist. - 2013. Vol. 18(6). P. 707-716.

18. Sequist L.V., YangJ.C., Yamamoto N., O'Byrne K., Hirsh V., Mok T., Geater S.L., Orlov S., Tsai C.M., Boyer M., Su W.C., Bennouna J., Kato T., Gorbunova V., Lee K.H., Shab R., Massey D., Zazulina V., Shabidi M., Schuler M. Phase III study of afatinib or cisplatin plus pemetrexed in patients with metastatic lung adenocarcinoma with EGFR mutations. // J Clin Oncol. - 2013. - Vol. 31(27). P. 3327-3334.

19. Shaw A.T., Ou S.H., Bang Y.J., Camidge D.R., Solomon B.J., Salgia R., Riely G.J., Varella-Garcia M., Shapiro G.I., Costa D.B., Doebele R.C., Le L.P., Zheng Z., Tan W., Stephenson P., Shreeve S.M., Tye L.M., Christensen J.G., Wilner K.D., Clark J.W., Iafrate A.J. Crizotinib in ROS1-Rearranged Non-Small-Cell Lung Cancer. // N Engl J Med. - 2014. Vol. 372(7). P. 683-684.

20. Stutman 0 . Tumor development after 3-methylcholanthrene in immunologically deficient athymic-nude mice. // Science. - 1974. - Vol. 183(4124). P. 534-536.

21. Temel J.S., Greer J.A., Muzikansky A., Gallagher E.R., Admane S., Jackson V.A., Dablin C.M., Blinderman C.D., Jacobsen J., Pirl W.F., Billings J.A., Lynch T.J. Early palliative care for patients with metastatic non-small-cell lung cancer. // N Engl J Med. - 2010. - Vol. 363(8). P. 733-742.

\section{References}

1.McGranaban N., Swanton C. Clonal Heterogeneity and Tumor Evolution: Past, Present, and the Future. Cell. 2017; 168: 613-628. doi: 10.1016/j.cell.2017.01.018. PMID: 28187284.

2. Lynch T.J., Bell D.W., Sordella R. et al. Activating mutations in the epidermal growth factor receptor underlying responsiveness of non-small-cell lung cancer to gefitinib. N Engl J Med. 2004; 350: 2129-2139. doi: 10.1056/ NEJMoa040938. PMID: 15118073.

3. Rolfo C., Giovannetti E., Hong D.S. et al. Novel therapeutic strategies for patients with NSCLC that do not respond to treatment with EGFR inhibitors. Cancer Treat Rev. 2014; 40: 990-1004. doi: 10.1016/j.ctrv.2014.05.009. Epub 2014 Jun 6. PMID: 24953979.

4. Sequist L.V., YangJ.C., Yamamoto N. et al. Phase III study of afatinib or cisplatin plus pemetrexed in patients with metastatic lung adenocarcinoma with EGFR mutations. J Clin Oncol. 2013; 31: 3327-3334.

5. Couzin-Frankel J. Breakthrough of the year 2013. Cancer immunotherapy. Science. 2013; 342: 1432-1433. doi: 10.1126/science.342.6165.1432. PMID: 24357284.

6. McCarthy E.F. The toxins of William B. Coley and the treatment of bone and soft-tissue sarcomas. Iowa Orthop J. 2006; 26: 154-158. PMID: 16789469.

7. Stutman 0 . Tumor development after 3-methylcholanthrene in immunologically deficient athymic-nude mice. Science. 1974; 183: 534-536.

8. Hunig T. T-cell function and specificity in athymic mice. Immunol Today. 1983; 4: 84-87. doi: 10.1016/01675699(83)90125-1. PMID: 25291286.

9. Burnet M. Cancer; a biological approach. I. The processes of control. Br Med J. 1957; 1: 779-786. PMID: 13404306.

10. Chen W. IDO: more than an enzyme. Nat Immunol. 2011; 12: 809-811.

11.Jobnson D.B., Peng C., Sosman J.A. Nivolumab in melanoma: latest evidence and clinical potential. Ther Adv Med Oncol. 2015; 7: 97-106. doi: 10.1177/1758834014567469. PMID: 25755682.

12. Reck M., Rodriguez-Abreu D., Robinson A.G. et al. Pembrolizumab versus Chemotherapy for PD-L1-Positive Non-Small-Cell Lung Cancer. N Engl J Med. 2016; 375: 1823-1833. 
13. Gonzalez-Angulo A.M., Morales-Vasquez F., Hortobagyi G.N. Overview of resistance to systemic therapy in patients with breast cancer. Adv Exp Med Biol. 2007; 608: 1-22. PMID: 17993229.

14. Bergethon K., Shaw A.T., Ou S.H. et al. ROS1 rearrangements define a unique molecular class of lung cancers. J Clin Oncol. 2012; 30: 863-870.

15. Camidge D.R., Bang Y.J., Kwak E.L. et al. Activity and safety of crizotinib in patients with ALK-positive nonsmall-cell lung cancer: updated results from a phase 1 study. Lancet Oncol. 2012; 13: 1011-1019. doi: 10.1016/S14702045(12)70344-3. Epub 2012 Sep 4. PMID: 22954507.

16. Shaw A.T., Ou S.H., Bang Y.J. et al. Crizotinib in ROS1-Rearranged Non-Small-Cell Lung Cancer. N Engl J Med. 2014; 372: 683-684.

17. Temel J.S., Greer J.A., Muzikansky A. et al. Early palliative care for patients with metastatic non-small-cell lung cancer. N Engl J Med. 2010; 363: 733-742. doi: 10.1056/NEJMoa1000678. PMID: 20818875.

18. Goldberg R.M. N9741: a phase III study comparing irinotecan to oxaliplatin-containing regimens in advanced colorectal cancer. Clin Colorectal Cancer. 2002; 2: 81.

19. Bass A.J., Thorsson V., Shmulevich I., Reynolds S.M. Comprehensive molecular characterization of gastric adenocarcinoma. Nature. 2014; 513: 202-209. doi: 10.1038/nature13480. Epub 2014 Jul 23. PMID: 25079317.

20. Cerami E., Demir E., Schultz N., Taylor B.S., Sander C. Automated network analysis identifies core pathways in glioblastoma. PLoS One. 2010; 5: e8918. doi: 10.1371/journal.pone.0008918. PMID: 20169195.

21. Rooney M., Devarakonda S., Govindan R. Genomics of squamous cell lung cancer. Oncologist. 2013; 18: 707-716. doi: 10.1634/theoncologist.2013-0063. Epub 2013 May 31. PMID: 23728941. 